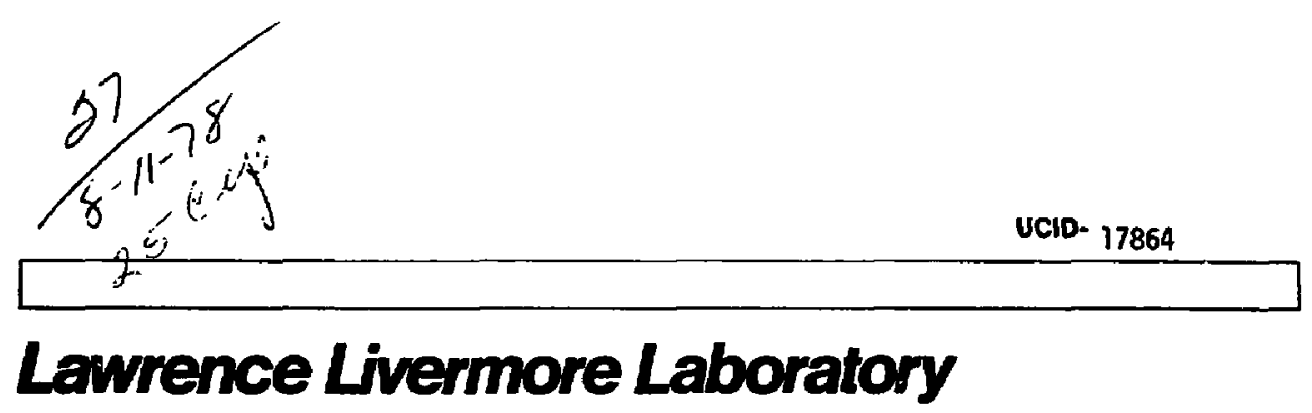

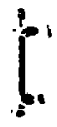

\title{
A STUDY OF EUV EMISSIONS FROM 2XIIB
}

R.P. Drake, K.I. Chen, H.W. Moos, J.L. Terry

The Johns Hopkins University and

R.S. Hornady

Lawrence Livemore Laboratory

June 27, 1978
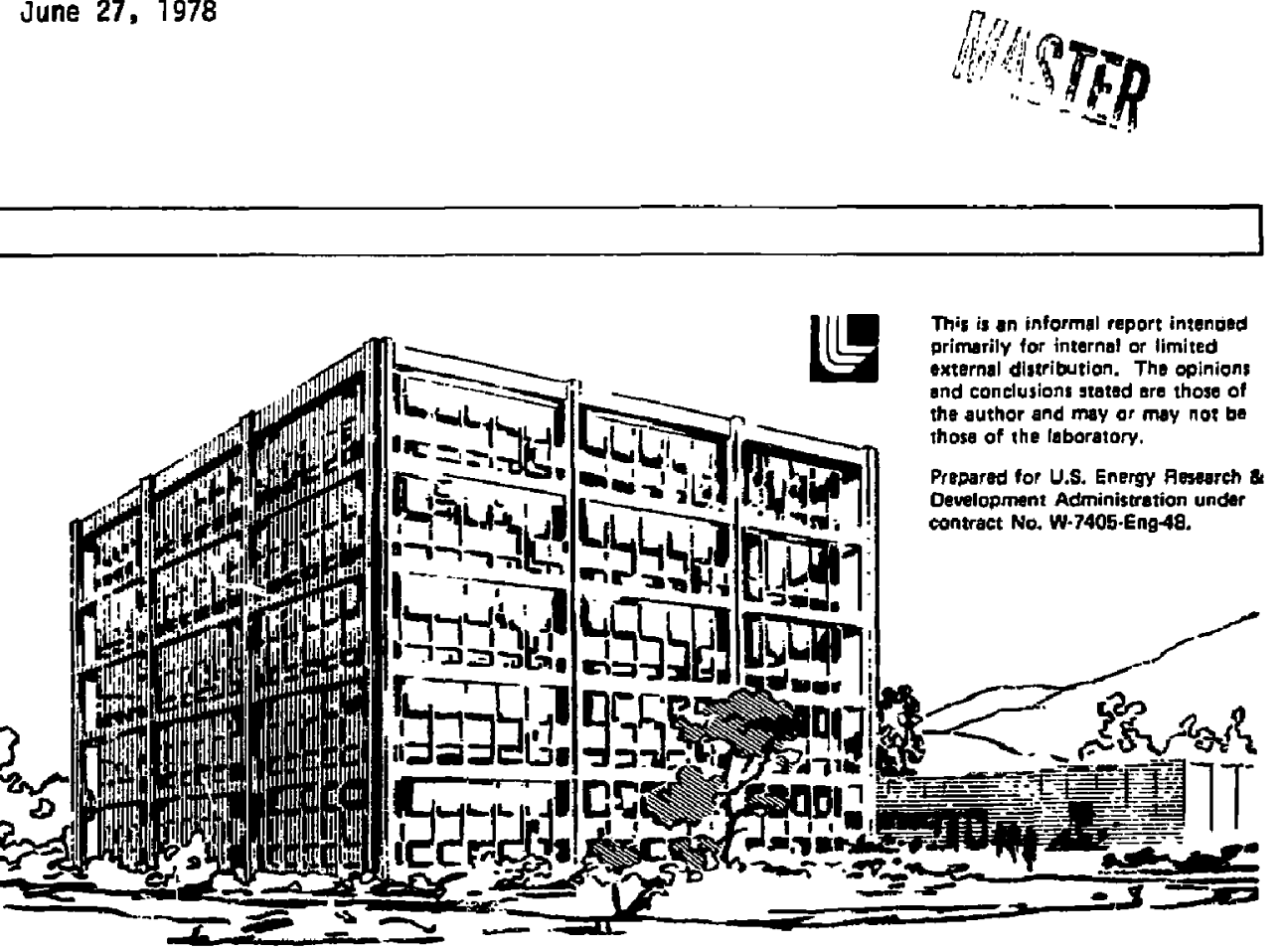

$+$

DISTRIBUTION OF TUS DOCUMENT IS UNLLMTED 


\title{
A STUDY OF EUV EMISSIONS FROM 2XIIB*
}

\author{
R.P. Drake, K.1. Chen, H.W. Moos, J.L. Terry \\ The Johns Hopkins University
}

\author{
R.S. Hornady
}

Lawrence Livermore Laboratory

\section{ABSTRACT}

Initial results from a study of EUV emissions from the $2 \times 118$ experiment are reported. Time-dependent brightness measurements of deuterium, oxygen, nitrogen, carbon and titanium emissions from the central 2 XII 8 plasma have been made. The deuterium Lyman alpha brightness is $1.5 \times 10^{17}$ photons $\mathrm{sec}^{-1} \mathrm{~cm}^{-2} \mathrm{sr}^{-1}$. The Lyman alpha time development and broad spectral profile are consistent with other knowledge of the 2XIIB plasma. Oxygen is the dominant impurity in 2XIIB. The 0 VI $1032 \&$ brightness is $1.65 \times 10^{18}$ photons $\mathrm{sec}^{-1} \mathrm{~cm}^{-2} \mathrm{sr}^{-1}$. The oxygen concentration is $2 \%$ and its estimated confinement lifetime is j00 $\mathrm{HS}$; this may jmply mirror confinement of the oxygen ions. Nitrogen and carbon concentrations are $0.4 x$ and $0.3 x$, respectively. It is not certain whether these impurities are mirror confined. The titaniun concentration is believed to be low compared to that of oxygen. The power loss from the central plasma due to radiation by and ionization of light impurities is $260 \mathrm{kH}$, which is $4 \times$ of the power deposited by the neutral beans. Further studies of impurity sources and penetration are now being performed.

«Performed under the auspices of the U.S. Department of Energy by the Lawrence Livermore Laboratory under contract number W-7405-EHG-48 and by the Johns Hopkins University under contract number EY76-5-02-?711.

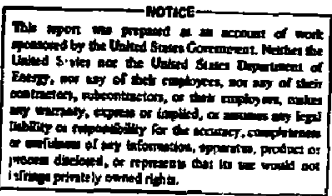




\section{Page}

I. Introduction 2

II. Basic Concepts of EUV Diagnostics 4
A. Brightnesses 4

B. Lifetimes and Rates 5

C. Impurity Densities and Concentrations 10

D. Impurity Currents and Fluxes 11

III. Experimental Details 12

A. Spectrometer System 12

B. 2XIIB Pa:ameters and the Spectrometer Installation $\quad 15$

IV. EUV Emission Characteristics 16

A. Observed Ions and Emission Characteristics 16

B. Absolute Brightness Measurements 20

C. Machine Conditions for these Measurements 22

D. Titanium Washer Gun Observations 26

v. Power Loss Due to Light Impurities 26

A. Theory of Spectroscopic Power Loss

B. Radiated Power Loss Results 30

C. Additional Impurity Power Loss 30

D. Implications of 2 XIIB Power Loss 32

VI, Light Impurity Results 33

VII. Lyman Alpha Measurements 36

vIII. Conclusion $\quad 42$

Acknowledgements $\quad 44$

References $\quad 45$ 


\section{A STUDY OF EUV EMISSIONS FROM $2 X I I B$}

\section{INTRODUCTION}

This report describes an extreme ultraviolet (EUV) spectroscopic study of impurities in the neutral beam heated mirror machine, 2XIIB. As in all high temperature plasma devices, issu.rs such as impurity power loss and the effects of impuritles on the $T_{e}$ proffle are of concern. On the one hand, mirror machines in particular are not expected to have an impurity problem, as they are open-ended. High $Z$ impurities sca:ter into the loss cone fastor than deuterium ions do. In addition, the ambipolar plasma potentiz: should preferentially eject impurities, and should prevent their penetration from the ends. On the other hand, the success of stream stabilization of 2XIIB ${ }^{1}$ raisas questions about the effectiveness of the ambipolar potential in excluding particles, including impurities, frum the plasma. In addition, impurities in the high energy neutral beams may be injected and confined despite the plasma potential. Furthermore, impurities may play a beneficial role, by means of the "Ohkawa Current", 2 in field reversal experiments. This occurs because electron currents may cancel the ion currents which reverse the magnetic field, but impurities can impede the electron currents by increasing $Z_{\text {eff }}$.

In view of the above considerations, a study of impurities in a machine of this type is of interest. In cooperation with the Lawrence Livermore Laboratory, the Johns Hopkins University is investigating the nature and implications of EUV emissions from the central $2 \times I I B$ plasma. The time-dependent emissions from the dominant species $D$, light impurities 10 , C, and $N$ ) and titanium are being measured with high photometric accuracy. Data of this type can be used to address questions such as impurity 
concentrations, confinement, lifetimes, sources, transport, and power loss. This report is a presentation of the initial results of that experiment. obtained during a study of the 2XIIB EUV emissions, conducted from September 19 to Decenber 22, 1977. Some knowledge gained in early 1978 is used to interpret the data.

At the outset of this study, a surprising result was that the EUY emissions from 2XIIB are very bright, even in comparison with tokanak emissions. The oxygen concentration is large and emits substantial quantities of radiation from highly ionized states. The high power loss, due to radiation and ionization, is more than $10 \mathrm{~W} \mathrm{~cm}^{-3}$. Although this power loss is a low percentage of the deposited neutral beam power, it may have implications for the larger, less intensely heated mirror experiments of the future. These and other results from this initial study are covared in this report.

The quantities presented and evaluated in this report are defined and discussed in Section II. Section III of this report is a brief description of experimental details, including the JHU spectrometer and $2 \times 118$ parameters. Section IV reports the impurity measurements. The identified spectes and the measured brightnesses are presented. The time histories of impurity emissions are described, and the machine conditions for this data are discussed. Section $V$ considers the question of power loss due to impurities, and Section VI presents the estimates of impurity confinement times, concentrations, and currents wich mav be obtained from the data. Section VII relates the brightness and spectral profile of the dueterium Lyman alpha entissions to the velocity distribution of the mirror confined fons and atoms from the neutral bean and scrrounding ga,. A summary and conclusions are presented in Section VIII. 
II. BASIC CONCEPTS OF EUV DIAGNOSTICS

This section discusses the relationship of the impurity concentrations, fluxes, and confinement times to the measured EUY brightness. Note that these relationships, which are used in Section VI to compute these quantities from the empirical brightness data, are model dependent; the relfability and details of these models are improving with continued study of 2 XIIB.

\section{A. Brightnesses}

The measured brightness of an emission line from an optically thin source, is given by

$$
B=\frac{1}{4 \pi} \int E(\underline{r}) \mathrm{d} 1 \text { photons } \mathrm{sec}^{-1} \mathrm{~cm}^{-2} \mathrm{sr}^{-1} \text {, }
$$

Where $E(\underline{r})$ is the volume emission rate in photons $\mathrm{sec}^{-1} \mathrm{~cm}^{-3}$ into $4 \pi$ steradians and the integral is taken along the line of sight of the measuring instrument. $E(\underline{r})$ can be replaced by more microscopic quantities if one considers the cause of the photon emissions. The only important processes related to amission, in this type of plasma, are collisional excitation and radiative decay.* As radiative decay is much faster than collisional excitation, the emission rate is determined by the excitation rate, thus

$$
E=n_{e^{N G<\sigma} v>e x}^{e x}
$$

\footnotetext{
*Photon excitation is unimportant as 2XIIB plasmas are optically thin; collisional de-excitation is not significant because radiative decay is much faster.
} 
$\langle\sigma v\rangle^{\text {ex }}$ is the rate coefficient for electron impact excitation, ${ }^{\star} G$ is the branching ratio for the transition being measured $G=1$ for resonance transitions), $N$ is the density of ions in the lower state of the transition, and $n_{e}$ is the electron density.

One can now obtain a relationship between the measured monochromatic surface brightness of the plasma and the microscopic electron and impurity ion densities. The substition of (2) into $(1)$ yields

$$
\left.B=\frac{1}{4 \pi} \int n_{e} N<\sigma v\right\rangle^{e x} d 1
$$

The transitions observed have energies on the order of $15 \mathrm{eV}$. In such cases, <oy ${ }^{e x}$ varies slowly with $T_{e}$ above $20 \mathrm{eV}$. In consequence, <or ${ }^{\mathrm{ex}}$ can usually be withdrawn from the integral in this study. The best available excitation rate coefficients were used ${ }^{3-7}$ to obtain the impurity concentrations. *

\section{B. Lifetimes and Rates}

The rate, $\nu$, and lifetime $\tau$, are defined by

$$
v=\frac{1}{\tau}=\frac{1}{x} \frac{d x}{d t} ;
$$

if $\tau$ is not a function of $x$, it is the time for exponential growth and decay of the quantity $x$. For collisional excitation, as described above, $v=$ $n_{e}<\sigma v>^{e x}$ and is typically $10^{6} \sec ^{-1}$. For radiative decay $v$ is the radiative spontanecus enission rate, on the order of $10^{9} \mathrm{sec}^{-1}$ for the transitions measured here. These rates provide clear justification for the model of Eq. (3).

tIon impact excitation is small compared to the uncertainty in <ov> , as long as the ion velocity is not comparable to the electron velocity. **Refs. 3-7 generally agree quite well on these values. In cases of disagreement, the close coupling calculations of Robb (3) were used. 
A number of atomic collision processes must be considered to understand the behavior of impurities in a plasma. Lifetimes for several processes, computed using $2 X I I B$ parameters, are given in Table II-1 for the important ionization states of oxygen. Table II-2 presents the formulas and references used to compute these lifetimes. ${ }^{4,8-13}$ Electron impact ionization is very fast for oxygen ions of low charge in the central $2 \times 1$ IB plasma, but is less rapid for highly charged lons. Charge exchange processes,

$$
0^{2}+0^{0}+0^{2-1}+0^{+}
$$

are important in this plasma because of the relatively large density of high energy neutrals. Charge exchange uttimately limits the population of high ionization states. In contrast, the low electron density of this plasma implies that recombination is negligible. The Spitzer drag and $90^{\circ}$ scattering times given in Table II -1 imply that a high energy impurity may be confined for a few hundred microseconds, while a low energy impurity should not be confined at all. Additional processes which are still under investigation, such as deuteron impact ionization, may also affect the behavior of impurities in $2 \times I I B$. Such processes will not be considered here.

One of the goals of this study was determination of the confinement lifetime of impurity ions in the 2 XIIB plasma. The best way to determine the lifetime of observed inpurities is with an accurate computer model of the plasma, including transport and using good rate coefficients. As a next approximation, a zero dimensional code can simulate transport by the use of confinement times. By successfully modeling the time behavior of impurity emissions, using known $T_{e}(\underline{r}, t)$ 
THALE :1-1

Inpurity Time scales*

\begin{tabular}{|c|c|c|c|c|c|c|c|c|}
\hline \multirow[t]{2}{*}{ PROCESS } & \multicolumn{7}{|c|}{ LIFETIYE OF SIAIE FOR PROCESS IN MIGROSECOONSS } & \multirow{2}{*}{ REF. } \\
\hline & ot & alt & olli & oIV & oy & ovi & ovit & \\
\hline Ionizationtt & 0.24 & 0.84 & 2.4 & 6.4 & 22 & $n$ & $3.3 \times 10^{5}$ & (8) \\
\hline Charge Exchange & 0.29 & 270 & 910 & $\mathbf{3 3}$ & 40 & 50 & 63 & $\{9,10,21\}$ \\
\hline Spltzer Drag & & 6700 & 1700 & 750 & 420 & 270 & 190 & (12) \\
\hline Reconbination & & $1.7 \times 10^{5}$ & $3 \times 10^{4}$ & $2 \times 10^{4}$ & $4 \times 10^{4}$ & $2.5 \times 10^{5}$ & $1.2 \times 10^{5}$ & (4) \\
\hline \multicolumn{9}{|l|}{ Solf -5cattering Life. } \\
\hline time: 100 eV oxygen & & 1900 & 120 & 24 & 7.8 & 3.0 & 1.5 & (13) \\
\hline 10 kel oxygen & & $1.9 \times 10^{6}$ & $1.2 \times 10^{5}$ & $2.4 \times 10^{4}$ & $7.8 \times 10^{3}$ & $3.0 \times 10^{3}$ & $1.5410^{3}$ & \\
\hline \multicolumn{9}{|l|}{$\begin{array}{l}\text { Oxygen off Deuterium } \\
\text { Scattering Lilutine: }\end{array}$} \\
\hline 100 ev oxygen & & 1200 & 280 & 120 & 69 & 45 & 30 & \\
\hline 10 keV Oxygen & & $3,1 \times 10^{5}$ & $2.8 \times 10^{4}$ & $1.2 \times 10^{4}$ & $6.9 \times 10^{3}$ & $4.5 \times 10^{3}$ & $3 \times 10^{3}$ & \\
\hline
\end{tabular}


PROCESS

IONIZATION

CHARGE EXCHANGE

SPITZER DRAG

RECOMBINATION

SCATTERING $\tau=\frac{1}{n_{\mathrm{e}}<\sigma v>^{i o n}}$

$\operatorname{Lotz}^{8}$

Gardner 9

Cranda 170

ARML 11

REFERENCE

$\tau=\frac{1}{n_{0}\langle\sigma v\rangle c x}$ (ions) $\tau=\frac{10^{13} \mathrm{MT}_{\mathrm{e}}^{3 / 2}(\mathrm{keV})}{n_{\mathrm{e}} \ln \Lambda \mathrm{Z}^{2}}$

$I=\frac{1}{n_{e}<\sigma v>^{R E C}}$ $=\frac{1}{n_{e}<\sigma v{ }^{c x}}$ (neutraIs)

Spitzer ${ }^{12}$

Mattio $7 i^{4}$

This is scattering of species a off species b neglecting potential effects, with $E$ in keV and $A$ in Ams. 
and $n_{e}(\underline{r}, t)$ profiles, one can in principle obtain the impurity confinement times. Unfortunately, the rate coefficients and $T_{e}$ profiles needed are not precisely known. This is particularly true for charge exchange prucesses. In addition, consideration of the efferts of impurity transport leads to both complexity and uncertainty in the model. Despite these problems, one can easily make a factor-of-two estimate of the impurity lifet ime by crude methods.

Two methods of estimating impurity confinement times will be presented. First, if one knows the volume rate of influx, $c\left(\mathrm{~cm}^{-3} \mathrm{sec}^{-1}\right)$, of an impurity species such as oxygen, and the density of that species, then in steady state the average iffetime of that species is

$$
\tau_{c}=\frac{N}{C}
$$

where $N=\sum N_{j}$; the sum is over all ionization states. $C$ may be found by evaluating the , Jlume rate at which impur ity ions pass through some low ionization state in which, due to its rapid ionization, confinement losses are expected to te small. The significant processes resulting in the net upward flow of impurity ions into higher ionization states are ionization and charge exchange. Thus,

$$
C=N_{i} n_{e}<\sigma v>_{i}^{i o n}-N_{i+1} n_{0}<\sigma v>>_{i+1}^{c x}
$$

The volume rate of ionization is $N_{i} n_{e}\langle\sigma v\rangle_{i}^{i o n i z a t i o n ;} N_{i}$ is the density of impurities in the low ionization state determined from the EJV data, and $<0 v>i$ ion is the rate coefficient for electron impact ionization out of state $i$. The volume rate of charge exchange, is $n_{i+1} n_{0}\langle\sigma v\rangle_{i+1}^{c x} ; n_{0}$ is the neutral deuterium density and $\left\langle\sigma v{ }_{i+1}^{c x}\right.$ is the rate coefficient for charge exchange into state $i$. 
The secons method of estimating impurity lifetimes is simpler than the first. An impurity species must in general be confined long enough to reach the ionization state observed to be most populous. This time is the sum of the ionization times for each state below the most populous state, 2:

$$
\tau_{c}=\sum_{i<2} \tau_{i}=\sum_{i<2} \frac{1}{n_{e}\langle\sigma v\rangle_{i}^{i o n}}
$$

This value is a minimum estimate of the confinement time because it does not include the impurity iffetime in the most populous ionization state. $t$ may be significantly underestimated if the impurity confinement time in the terminal states is long compared to the ionization times considered.

\section{Impurity Densities and Concentrations}

If one knows $n_{e}(r), T_{e}(r)$ and the spatial profile of the impurity ions, then Eq. (3) can be integrated to obtain the impurity density of that ionization state. If this can be done for all ionization states of a given impurity species, the total density of that species and hence its concentration in the plasma are known.

As a practical matter, one does not know the impurity spatial profiles very we11, so one uses simple models, three of which will be mentioned here: the flat profile, the Gaussian profile, and the thin shell model. If the impurities are evenly distributed, and the $T_{e}$ profite is sufficiently broad, both $n$ and $<c$ v ex can be removed from the integral in (3), ylelding

$$
H_{i}=\frac{4 \pi B}{\left.\langle\pi v\rangle^{e x} / n_{e} d\right]}\left(\mathrm{cm}^{-3}\right)
$$

(Spatial scans conducted in early 1978 and Thomson scattering data indicate that this is the appropriate model for $2 \times 118$.$) In the second model, the$ $n_{e}$ and $n$ profiles are both approximately Gaussian ( and $\left.<0 v\right\rangle^{e x}=$ const.) and Eq. (3) can be integrated directly. Finally, if the impurity ions exist 
in a shell at $r=a$, Eq. (3) tecomes

$$
\left.B=\frac{1}{4 \pi} n_{e}(a) n<\sigma v\right\rangle^{e x} 2 \Delta w,
$$

where $\Delta w$ is the shell thickness.

\section{Impurity Currents and Fluxes}

The total number of impurities per second entering the plasma is the impurity current. This current, expressed in equivalent anperes, is

$$
I=e \int \frac{N}{T} d V \text {. }
$$

The impurity current is useful for comparison with known sources of deuterium. The current calculated in this report is that entering the central plasma, given by

$$
I=\frac{\text { eNV }}{\tau_{c}}=\frac{\text { eN } \pi R_{p}^{2} L}{\tau_{c}} .
$$

The value of $\mathrm{N}$ used in Eq. (11) should be consistent with the densities and population distributions involved in the estimation of ${ }^{\tau_{c}}$ by Eq. (5) or Eq. (7). The relation of the current to the average impurity flux is

$$
\Gamma=\frac{1}{A} .
$$

$A$ is the area over wich the impurity current enters the plasma. A good discussion of impurity fluxes and their estimation is presented in Ref. 14.

The impurity flux may be directly estimated for inward moving impurities wich form concentric shells of increasing ionization state as the radius decreases and the temperature increases in a sheath. If the impurities ionize faster than they diffuse or charge exchange, the flux at the radius 
is 14

$$
\Gamma=2 \pi B \frac{<a v z^{\text {ionization }}}{<\sigma v\rangle^{e x}}
$$

Eq. (13) may be seen to represent the impurity flux as the photon flux $(4 \pi B)$ divided by the average number of photons emitted per ion ( $<$ ov $\rangle^{\text {ex }} /\langle 0 \mathrm{v}\rangle^{\text {ion }}$ ) for a shell observed twice along the line of sight. If the impurities enter the plasma by another means, such as neutral beam injection, Eq. (13) does not apply. However, the implication of Eq. II-(8), (11), and (12) is that 2 high value of the brightness implies a high concentration, and hence a large impurity flux or/and a long confinement time.

\section{EXPERIMENTAL DETAILS}

The JHU spectrometer has been described in detail elsewhere, ${ }^{15}$ as has the 2 XIIB plasma device..$^{16}$ This section contains a brief description of the spectrometer, the plasma device and the experimental setup at 2 XIIB. The orientation of the spectrometer is shown in Fig. III-1. Figure III-2 exhibits data indicating some of the 2 XIIB plasma characteristics.

\section{A. Spectrometer System}

The instrument is a $0.4 \mathrm{~m}$ normal incidence extreme ultraviolet monochromator. It utilizes a 2400 line/mm platinum coated grating blazed at $300 \AA$, and a windowless photomultiplier tube with a CU I photosensitive surface to obtain a spectral range of $300 \AA$ to $1700 \AA$; the time resolution is 70 microseconds. Typical slit settings were $2 \&$ (FWHM) to completely cover the spectral line width.

The instrument has been absolutely calibrated at the National Bureau of Standard's (on the SURF-II synchrotron radiation source) and at JHU using HBS diode detectors as the calibration reference. 17 These calibrations agree. The estimated total uncertainty in the absolute sensitivities was 


\section{JHU Monochromator}

Thoinson scattering

\section{Ruby laser}

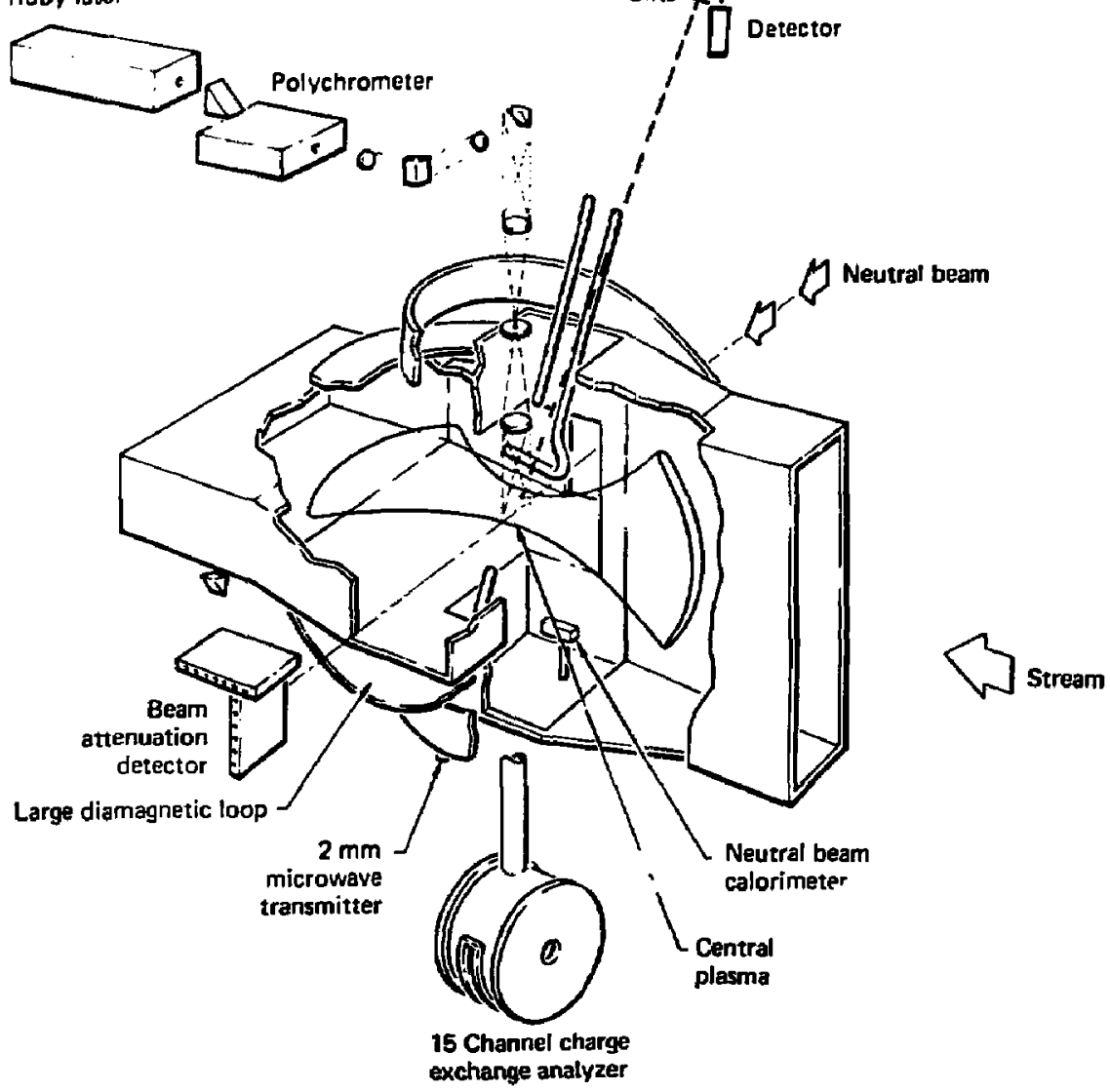

Figure III-1. Schematic of selected 2XIIB diagnostics, inciuding EUV monochrometer system. 
1
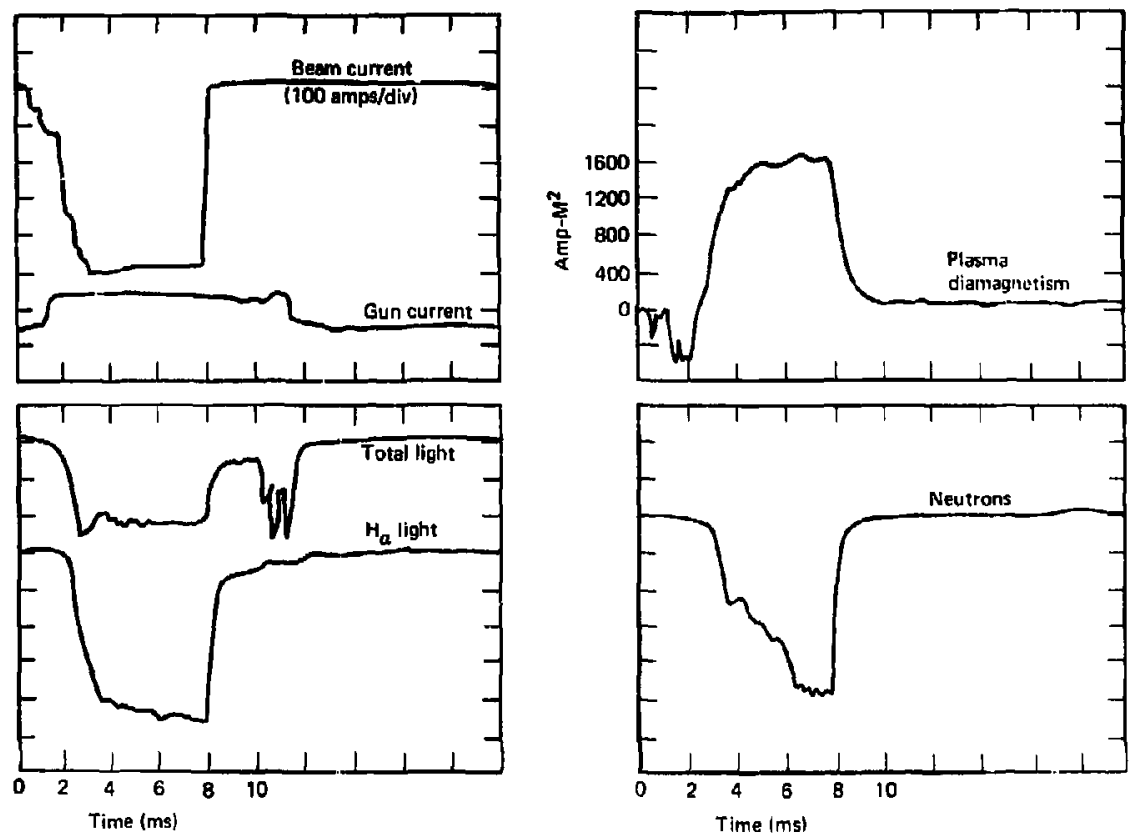

Figure II -2. 2XIIB diagnostic traces from shot 25 oct. 24, 1977. 
less than $+25 \%$. This value is subject to drifts in the calibration, which will be determined at the end of the present experiment. The monochromator is equipped with a preslit, $40 \mathrm{~cm}$ from and parallel to the entrance slit, which allows selective variation of the widih and center of the field of view; an $\mathrm{MgF}_{2}$ window enables elimination of second order radiation above 1150 R.

Some special preparations were made for the $2 x I I B$ experiment. The spectrometer slits were recalibrated, and the absolute sensitivity calibration was repeated. New maynetic shielding was sesigned, constructed, and tested to protect the photomultiplier from the 500 gauss flelds near 2XIIB.

\section{B. 2XIIB Paraneters and the Spectrometer Installation}

$2 \times I 1 B$ is a neutral beam heated minimum-B geometry plasma confinement device. $^{16}$ For the oxygen results obtained October 24 , which are emphasized in this report, it produced high beta (beta $>1$ ) plasmas in a 6.7 KG central vacuum field. The electron temperature was typically $150 \mathrm{eV}$; the ion temperature was approximately $13 \mathrm{keV}$. Line densities were about $1.5 \times$ $10^{15} \mathrm{~cm}^{-2}$, with a 7 to $10 \mathrm{~cm}$ radius and a Gaussian radial profile implying a peak density of $1 \times 10^{14} \mathrm{~cm}^{-3}$. The hot plasma had a 15 to 18 $\mathrm{cm}$ l/e axial halfwidth.

The JHU instrument is positioned on top of $2 \times 118$ and looks down through the yin yang coils into the midplane region. The entrance slit is 2.8 in from the machine center, about 13 degrees north and 3 degrees east of the vertical axis. The line-of-sight is through the hot plasma in the center of 2XIIB (See Fig. 11-1). The field of vien is typically $1 \mathrm{~cm}$ radially by $3 \mathrm{~cm}$ along the magnetic axis. The spatial alignment of the spectrometer was tested by scanning the plasma, and was found to be approximately correct. 


\section{EUV EMISSION CHARACTERISTICS}

The basic experimental data, which will be further analyzed later, are presented in this section. The observed impurity ions ane ?ieted; a!? ? described are the time develcpment and aisolute brightnesses of the impurity enissions. Brightness measurements of both the target plasma formed by washer stack guns and the hot plasma established by neutral beam injection are reported, as we 11 as the machine conditions during these experiments. I brief discussion of the washer guns as an impurity source is given in subsection $D$.

\section{A. Observed lons and Emission Characteristics}

Spectral emission lines due to ionization states of oxygen, nitrogen, carbon, and titanium have been observed. The lines observed are tabulated in Table IV-T, which includes an indication of our confidence in their identification. This corfidence level is based on observation of the transitions from another plasma with the same instrument, and our ability to scan the spectral region, given a limited number of shots at constant machine parameters. The observed transitions are indicated; they are all ground state resonance transitions. Typical time histories of five oxygen states are presented in Figs. IV-I and IV-2.

The time deveopment of the $2 X I I B$ discharge can be divided into three phases: The target plasma buildup, the hot plasma buildup, and the high density plateau. During these experiments, a target plasma was provided by titanium washer guns beginning at 1.2 ms after machine "zero time". The hot plasma buildup began when the neutral beams fired at $1.8 \mathrm{~ms}$ and ended when the high density plateau was reached at about $4 \mathrm{~ms}$. The plateau lasted until about $8 \mathrm{~ms}$, depending on the duration of the neutral beam current.

During the target plasma buildup, low intensity impurity emissions were observed from low ionization states. Emissfons of D II and D III were 
TABLE IV- 1

Observed Ions and Transitions

\begin{tabular}{|c|c|c|c|c|}
\hline Ion & $\lambda(A)$ & Transition & & Confidence \\
\hline $\mathrm{Hl}$ & $1216\left(\mathrm{~L}_{u}\right)$ & $1 s-2 p$ & ${ }^{2} s-{ }^{2} p$ & A \\
\hline 0 VI & 1032 & $2 s-2 p$ & ${ }^{2} s-{ }^{2} p$ & A \\
\hline $0 V$ & 630 & $2 s^{2}-2 s 2 p$ & $1_{p}$ & A \\
\hline OIV & 554 & $2 s^{2} 2 p-2 s 2 p^{2}$ & ${ }^{2} p-{ }^{2} p$ & A \\
\hline $0 \mathrm{III}$ & 703 & $2 s^{2} 2 p^{2}-2 s 2 p^{3}$ & $3 p$ & A \\
\hline $0 \mathrm{Il}$ & 539 & $2 p^{3}-2 p^{2} 35$ & ${ }^{4} S$ & A \\
\hline C IV & 1548 & $2 s-2 p$ & ${ }^{2} s-{ }^{2} p$ & A \\
\hline C III & 977 & $2 s^{2}-2 s 2 p$ & ${ }^{1} p$ & A \\
\hline$N y$ & 1239 & $2 s-2 p$ & ${ }^{2} s-{ }^{2} p$ & A \\
\hline N III & 685 & $2 s^{2} 2 p-2 s 2 p^{2}$ & $2 p-{ }^{2} p$ & A \\
\hline Ti VII & 522 & $3 s^{2} 3 p^{4}-3 s 3 p^{5}$ & $3 p-3 p$ & C \\
\hline Ti TV & 779 & $3 d-4 p$ & & B \\
\hline
\end{tabular}

All transitions are ground state resonance transitions.

Confidence Key:

$A-G O O D$ Observed and scanned here and elsewhere with this equipment.

B-GOOD observed and scanned in this study only.

C-POOR Incompletely scanned in this study only. 


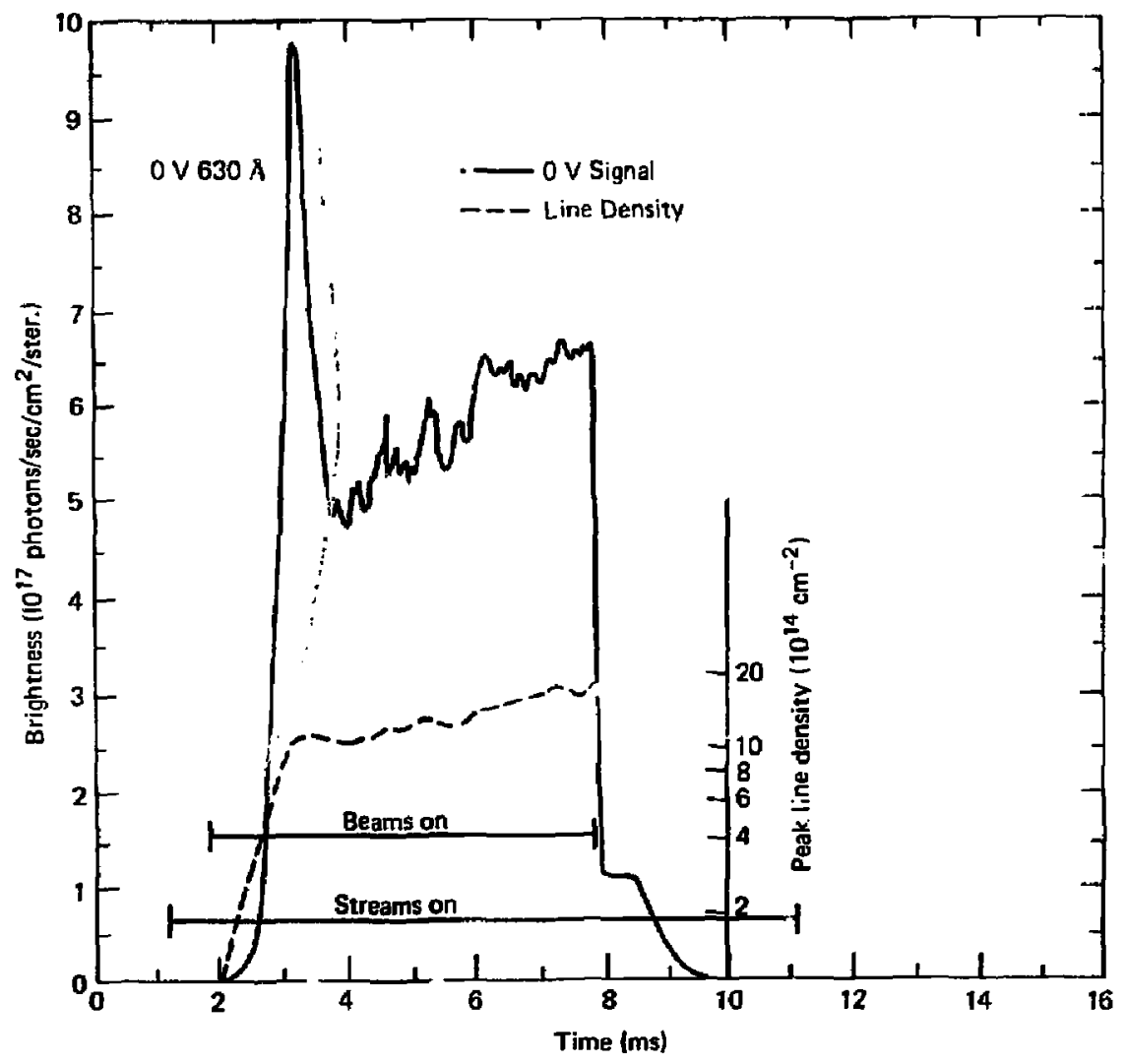

Shot 25 10/24/77

Parameters at $6 \mathrm{~ms}$

$T_{e}$

Radius

Line Density

Magnetic Moment

Neutral Beam Current

Mirror Ratio
$175 \mathrm{eV}$

$13 \mathrm{KeV}$

$9.5 \mathrm{~cm}$

$1.5 \times 10^{15} \mathrm{~cm}^{-2}$

1600 Amp-m $^{2}$

510 Amps

2

Figure IV-1. A typical o V time history, including early emission peak. Some plasma parameters and bean and stream durations are also shown. The dotted line is the line density time history. 


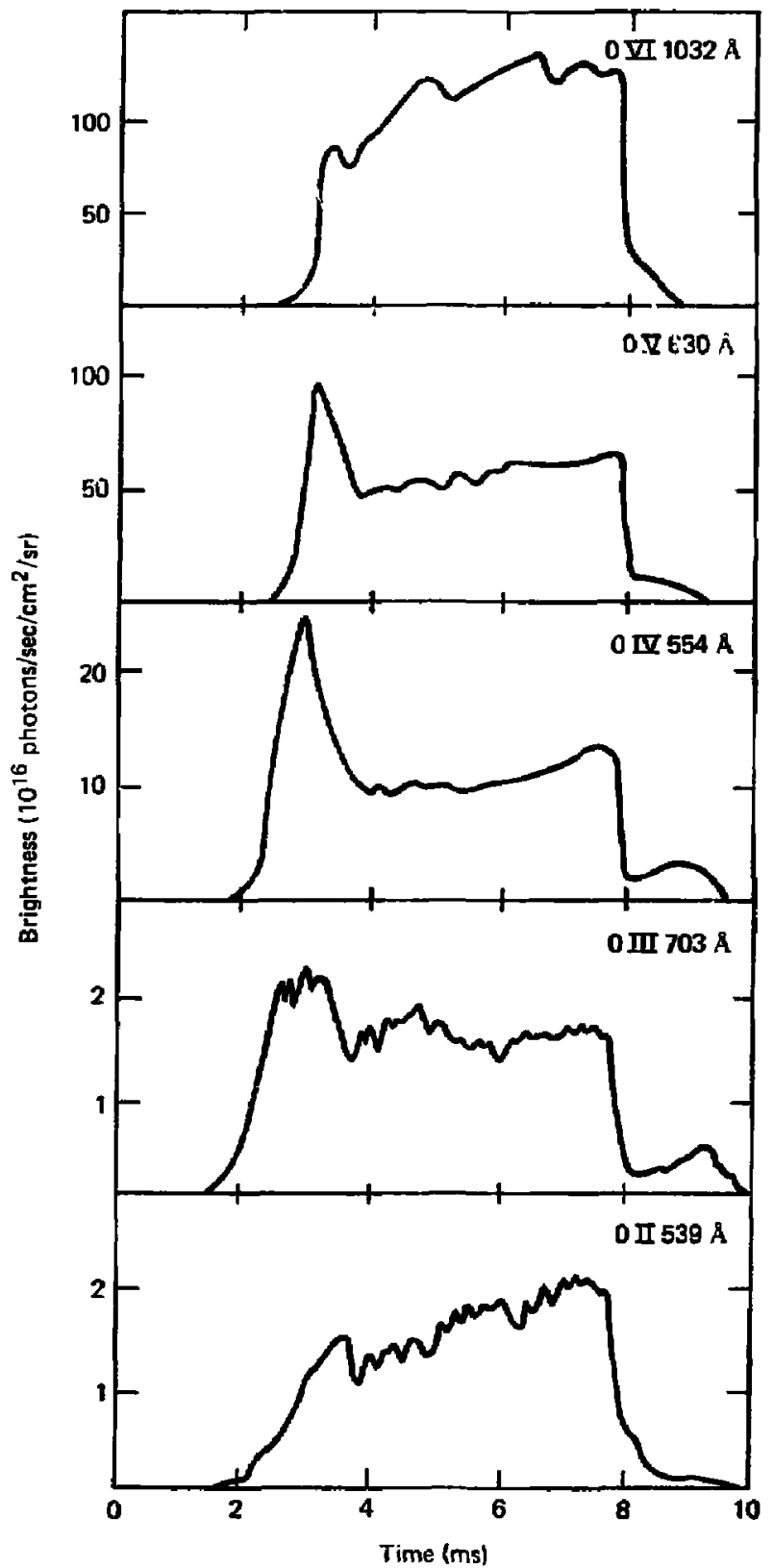

Figure IV-2. Typical emissions or the observed oxygen ions, from a series of identical shots on October 24, 1977. 
observed by $1.4 \mathrm{~ms}$, and 0 IV was observed by $1.8 \mathrm{~ms}$. The emissions at this tine were so dim as to require measurement on a more sensitive scale than that shown in Figure IV-2.

A strong increase in EUV impurity emissions occured during the hot plasma buildup. Brigintness peaks were observed from $0 \mathrm{IV}, 0 \mathrm{~V}$, and sometimes $0 \mathrm{Vt}$. These are belfeved to have resulted from the ionization of 0 II and 0 III fons already existing in the target plasma.

Impurity emissions showed some variations during the high density plateau. Emisstons from low ionization states often increased during this period, while emissions from higher ionization states exhibited variable changes. These variations were usually less than a factor of two.

\section{B. Absolute Brightness Measurements}

The absalute brightness (photons $\mathrm{sec}^{-1} \mathrm{~cm}^{-2} \mathrm{sr}^{-1}$ ) emitted from the plasma is the quantity directly measured by the EUV instrument. A summary of the brightnesses during the high density plateau is presented in Table IV-2.

Several factors contribute to the uncertainties in these measurements. The obvious factors of calibration accuracy and shot-to-shot vartation are considered. In addition, the effects of doppler broadening and background light levels are taken into account. Doppler broadened lines mere observed, but were not studied in detail.

The most striking fact is that EUV radiation from 2 XIIB is very bright. The OV and OVI brightnesses presented in Table IV-2 are more than two orders of lingnitude larger than those reported from Alcator, ${ }^{18}$ and an order of magnitude greater than those from TFR, ${ }^{14}$ yet those tokomaks have more than an order of magnitude greater deuterium conf inement time than $2 \times 11 B$. This indicates that the impurity fluxes or/and the confinement times are relatively high (See Section II-D). 


\section{TABLE IV-2}

High Density Plateau Brightnesses

Ion

(A)

Plateau Brightness

Brightness/<or $>^{\text {ex }}$

$$
\left(10^{16} \mathrm{ph} / \mathrm{sec} / \mathrm{crn}^{2} / \mathrm{ster}\right)
$$

$\left(10^{24} \mathrm{ph} / \mathrm{cm}^{5} / \mathrm{ster}\right)$

0 VI 1032 line

$165 \pm 65$

97

$0 \mathrm{~V}$

630 line

$70 \pm 26$

25

0 IV

554 multiplet

$10 \pm 5$

7.4

0 III 703 multiplet

$1.6 \pm 0.9$

1.6

0 II

539 multiplet

$1.05 \pm 0.55$

2.6

C IV 1548 doub 7 et

$11.6 \pm 6.7$

2.0

C III

977 line

$1.15 \pm 0.44$

0.18

N V

1239 line

$31.6 \pm 16$

14.1

N III 685 multiplet

$$
1.2 \pm 0.76
$$

0.49

Ti IV 779 line

$1.6 \pm 0.75$

Uncertainties include scatter in the data, calibration uncertainties, doppler broadening effects, and background light variations. These values apply to plasmas of Oct. 24, 1977 and to simtlar plasmas on other days.

*No reliable $\left\langle\sigma v>^{e x}\right.$ is avajlable. 
Table IV-2 also shows that oxygen is the dominant light impurity in 2XilB. The right hand column presents the measured brightnesses divided by the rate coefficients for excitation of these transitions. This quantity is proportional to the average density or these ions. These coefficients are relatively insensitive to $\mathrm{T}_{\mathrm{e}}$ above $20 \mathrm{eV}$, and were evaluated at $100 \mathrm{eV}$ for this calculation. It is quite clear that oxygen densities are greater than equivalent ion densities of other species. *

\section{Machine Conditions for These Measurements}

Impurity studies can be questioned if the data analyzed is not taken furing representative machine operation, or merely applies to a particiuar "bad" or "good" day. Such objections do not apply to this study, as wi11 be shown in this subsection.

The oxygen data analyzed here was taken on Oct. 24, 1977. This was a day of good machine operation; a description follaws. The purpose of this run was to observe the effects of cooling the neutral beam tank liquid nitrogen liners. Mo major changes were observed in plasma parameters or oxygen emissions as the liners cooled. Before the end of this run, but after the liners were cold, a number of shots were used to obtain the data p:esented in the prevfous subsection. The machine was gettered befcre each shot in the usual manner, and the getter wires were in good condition. The plasma parameters presented in Figures 111-2 and IV-1 are typical for this day, and show that this plasma was a normal one for this period.*t

*Ti densities are beljeved to be low on the basis of observations of $\mathrm{TI}$ VI and It VII during 1978.

**T $\mathrm{T}_{\mathrm{e}}$ vas typically $150 \mathrm{eV}$ for these shots, with $\pm 25 \mathrm{eV}$ variations from shot to shot. These variations in $T_{e}$ are not observed to effect EUV emissions or other plasma parameters. 
In addition, the oxygen transitions reported in Tables IV- 7 and IV-2 were all measured on at least one other day during October (with roughly comparable plasma parameters). In every such case the brightness was within a factor of two of that measured on October 24. Tak le IV-3 presents $0 \mathrm{~V}$ (630 \&) data from several days which span the period of this study. This table indicates that oxygen emissions do not vary greatly on a day-to-day time scale, and that the october 24 data is not unusual during that period.

The nitrogen and carbon data presented here were obtained on Nov. 2 and Nov. 3; the titanium data was obtauned Oct. 14. These runs were not atypical, a] though these measurements were made with lower beam cuirent and $\mathrm{T}_{e}\left(\mathrm{~T}_{e} \approx 70 \mathrm{eV}\right)$ than the oxygen measurements of 0ct. 24 . On Oct. 14 and Nov. 2 various stabilizing stream gun configurations were tried. The change in plasma parameters with a systematic variation of the neutral beam current during the Nov. 3 run showed that the plasma was similar to that studied over the preceeding six months. Carbon, nitrogen and titanium emissions had roughly comparable brightnesses on other days, although the data is not as extensive as that available for oxygen.

In summary, the oxygen data presented here was obtained under normal operating conditions, and there is no reason to suspect that the other impurity measurements are atypical.

\section{Titanium Washer Gun Observations}

Tab]e IV-4 presents the approximate brightnesses observed from plasmas created by a single 1/2" diameter washer gun, operated on a $2 \mathrm{~ms} 6$ to $8 \mathrm{kv}$ pulse line. These plasmas are quite variable, and hence only approximate brightness values are given. In this case the carbon light is much brighter than that of oxygen or nftrogen. This suggests that carbon is the dominant 
TABLE IV-3

Brightness of $0 \vee(630 \AA)$ on Various Days

$\begin{array}{lcll}\text { Date } & \text { Shot } & \begin{array}{l}\text { Brightness } \\ \text { ph sec }{ }^{-1} \mathrm{~cm}^{-2} \mathrm{sr}^{-1}\end{array} & \begin{array}{l}\text { Snedl } \\ (\text { Plateau })\end{array} \\ & & \mathrm{cm}^{-2} \\ 9 / 23 / 77 & 20 & 8.2 \times 10^{17} & 7 \times 10^{14} \\ 10 / 14 / 77 & 4 & 3 \times 10^{17} & 7 \times 10^{14} \\ 10 / 20 / 77 & 38 & 7.5 \times 10^{17} & 1.3 \times 10^{15} \\ 10 / 24 / 77 & 25 & 7.1 \times 10^{17} & 1.5 \times 10^{15} \\ 10 / 28 / 77 & 32 & 8.9 \times 10^{17} & 1.8 \times 10^{15} \\ 11 / 3 / 77 & 36 & 4.8 \times 10^{17} & 7 \times 10^{14} \\ 12 / 21 / 77 & 26 & 1.7 \times 10^{18} & 1.5 \times 10^{15} \\ 12 / 22 / 77 & 7 & 2 \times 10^{18} & 1.5 \times 10^{15}\end{array}$


TABLE IV-4

Streaming Plasma Observations

Ion

Wave-Tength

Approximate

Brightness

O II

$539 \AA$

$1 \times 10^{15}$

C III

$977 \AA$

$1.5 \times 10^{16}$

N III

$685 \AA$

$6 \times 10^{14}$

Ti IV

$799 \quad$ A

$>2 \times 10^{15}$

D I $\left(L_{\propto}\right)$

$1215.4 \AA$

$5 \times 10^{14}$ 
impurity from these washer guns, with the possible exception of Ti.* This canclusion was also reached in a study of similar plasmas performed on 2XII. 19

Every impurity species identified during beam-fueled discharges has been observed in washer gun plasmas. However, it is believed that the washer guns are not the only source of impurities on $2 \times 11 B$. The proportions of various impurities observed during beam-fueled discharges is quite different from those observed in the target plasma. (Compare Tables IV-2 and IY-4.) Furthemore, for oxygen, the impurity fluxes required to produce the observed 0 II brightnesses are much greater for beam fueled discharges than for washer gun created plasmas. Moreover, it is difficult to understand penetration of the plasma potential by quantities of cool streaming plasma impurities travelling along field iines. Further studies of 2XIIB wilt address the question of impurity sources in more detail.**

\section{PONER LOSS DUE TO LIGHT IMPURITIES}

Plasma impurities are of interest in part because they can radiate large

*More recent measurements suggest that titanium densities are greater than carbon densities in these plasmas. Titanium was excluded from 2 XII by time-of-flight trapping.

**Pulsed air leaks are not normally an impurity source. One such leak existed on $9 / 23 / 77$ and was jdentified and eliminated with the aid of the JHU spectrometer. Large increases in the nitrogen and oxygen signals were observed, beginning about one thermal transit time (from the vacuum chamber wall to the plasma) into the discharge. It is therefore clear that thermal gas, in sufficient quantities, can penetrate the $2 X I I B$ plasma. 
quantities of power. The absolute brightness measurements described above enable one to calculate the impurity power losses from 2XIIB. The method of calculation and the results for radiation, ionization and electron heating are presented below. The light impurity power loss is found to be about 63 $\mathrm{KW}$, which is $4 \%$ of the $1.5 \mathrm{MW}$ deposited by the neutral beams. This low percentage is due to the high density of energy deposition produced by neutral beam heating.

It must be emphasized that these results apply only to the light impurities $(0, C$ and $N)$ in the central plasma. The regions outside the central plasma contain several times as many particles, and may have a higher concentration of impurities producing a much larger radiation loss. While the effect of the impurities in the central plasma appears to be sma11, the total role of impurities in the 2 XIIB power balance is not clear.

\section{A. Theory of Spectroscopic Power Loss Measurements}

Spectroscopy provides an indirect measurement of power loss due to line radiation. The determination of power loss from absolute brightnesses is described in this section. Bolometric measurements are a desirable check on the completeness of these calculations, but such measurements have not been made on $2 X I I B$.

There is no universal relation between power loss and brightness. The measurad brightness results from an integration of light emitted along a line of sight through the plasma:

$$
B=\frac{1}{4 \pi} \int E(r) d l .
$$

Power loss by contrast results from the emission of light throughout the p]asma volume:

$$
P_{L}=h v \int E(r) d v \text {. }
$$


$P_{L}$ is the power radiated from the plasma by a spectral line with energy hu. Once the spatial variation of $E(r)$ is known one can relate power loss directly to brightness. Assuming cylindrical symmetry and a plasma of length $L$, one obtains

$$
\begin{aligned}
& B=\frac{1}{2 \pi} \int_{0}^{\infty} E(r) d r, \quad \text { and } \\
& P_{L}=2 \pi h v L \int_{0}^{\infty} E(r) r d r .
\end{aligned}
$$

Spatial scans of $2 \times 118$ (from early 1978) show that the radial profile of $E(r)$ is approximately Gaussian. Taking $E(r)=A e^{-r^{2} / a^{2}}$ we obtain:

$$
\begin{aligned}
& B=\frac{a}{2 \sqrt{\pi}} A \text { and } \\
& P_{L}=\pi h \cup L a^{2} A .
\end{aligned}
$$

Hence

$$
P_{L}=2 \pi^{3 / 2} h \nu L \text { a B }
$$

This gives the relation of power loss to measured brightness, assuming cylindrical symmetry and a Gaussian emission profile.

The total radiated power is desired, but Eq. (7) gives on ty the power radiated by an observed line. It is not practical to observe all radiating lines, so a calculation is necessary. It is necessary to relate the total power loss to the measured brightnesses. This relation is developed below. As $S(r)$ is proportional to cos $^{e x}$, the rate coeffecient for electron impact excitation of the transitions, one has:

$$
P_{L} a \frac{<\sigma y{ }_{L}^{e x}}{\lambda_{L}}
$$


The relation of this power to the power radiated by all lines of an ionization state, $P_{S}$, is

$$
\begin{aligned}
P_{S} & =F P_{L} \text {, where } \\
F & =\left[\sum_{i} \frac{\lambda_{L}}{\lambda_{i}} \frac{\langle\sigma v\rangle_{i}^{e x}}{\langle\sigma v\rangle_{L}^{e x}}+R_{m} \sum_{j} \frac{\lambda_{L}}{\lambda_{j}} \frac{\langle\sigma y\rangle_{j}^{e x}}{\langle\sigma v\rangle_{L}^{e x}}\right]
\end{aligned}
$$

The first sum is taken over the significant ground state transitions; and the second expression is summed over the important metastable transitions. The best values of $\langle\sigma y\rangle^{e x}$ exist for the transitions to the lowest excited states. The ratios of the other rate coefficients to these we 11 known ones (i.e., <ov $\rangle_{L}$ ) were estimated 20 using standard formulas 21 and the oscillator strengths of Wiese et al. 22 $R_{m}$ is the ratio of metastable populations to ground state population. By including all relevant proportionalities, $F$ converts the power radiated by the line into that radiated by the entire ionization state. Other ionization states may be included in $F$ in the same way the metastable system is in Eq. (9), but this is less desirable; in this study it was only necessary in the unimportant case of N IV.

The radiated power loss due to an entire species is then given by

$$
P_{\text {SPECIES }}=\sum P_{S}
$$

These uncertainties in these calculations have several sources. The absolute calibration of the spectrometer, the use of the spectrometer, the model relating radiated power to brightness, and the 
power factor $F$ all contribute to the uncertainty. However, as the relative populations of various ionization states need not be considered, this method is considerably more accurate than a purely a priori computation.

\section{B. Radiated Power Loss Results}

The power loss due to impurities was calculated as described atove. Brightness measurements have been made for all important ionization states of oxygen and for the significant states of carbon and nitrogen. Al1 $\Delta n=0$ and $\Delta n=1$ transitions were considered in evaluating the power factor. Metastable ratios were taken from measurements performed on Alcator, ${ }^{23}$ except for the important case of $0 \mathrm{~V}$, which was checked in the 2XIIB plasma. The emission radius was taken to be $9 \mathrm{~cm}$, and the length to be $15 \sqrt{\pi}$. The results of these calculations are presented in Table V-1.

Most of the power radiated by 2 XIIB impurities is from OV and OVI. This is due to the dominance of oxygen and to the fact that nitroyen and carbon reach helium-like states at lower temperatures than oxygen does.

\section{Additional Impurfty Power Loss}

Impurities also remove power from plasma electrons by means of ionization and electron heating. 14 This power loss depends on the rate of impurity influx, the maximum ionization state the impurities reach, and the number of cold electrons they produce in the plasma. The impurity influx and ionization are considered in Section VI. On the basis of those results, the ionization and electron heating power loss are found to be $20 \mathrm{~kW}$, with an upper limit of $62 \mathrm{kH}$. These losses are an appreciable fraction of the total impurity power loss, but are small compared to the input power to 2 XIIB. 


\section{TABLE V-1}

\section{LIGHT IMPURITY RADIATED POWER LOSS}

Spectes

Power Radiated

by Measured

Lines
Total Power

Radiated by

\begin{tabular}{ccc} 
OXYGEN & $15.6 \mathrm{kH}$ & $35.5 \mathrm{kH}$ \\
NITROGEN & $1.44 \mathrm{~kW}$ & $6.5 \mathrm{~kW}$ \\
CARBOH & $0.5 \mathrm{kH}$ & $0.6 \mathrm{kH}$ \\
\hline TOTAL & $18 \mathrm{kH}$ & $43 \pm 28 \mathrm{kH}$
\end{tabular}


Other more subtle processes may contribute to the impurity related power loss. For example, charge exchange processes in which deuterons gain electrons could lead to the loss of high energy particles from the plasma. These processes have not yet been considered in detail.

\section{Implications of 2XIIB Power Loss}

Apparently, the light impurities in the central 2XIIB plasma do not play a major role in determining its parameters. Neither $T_{e}$ nor the $T_{e}$ radial profile will be significantly affected by power losses such as these. The electron temperature limit due to impurity power loss may be estimated by equating this power loss to the Spitzer drag power input to the electrons from the hot ians. The power loss determined above allows a maximum $2 \times 1 I B$ electron temperature of about $500 \mathrm{eV}$, which is much higher than the observed values of $T_{e^{*}}$ One must conclude that if impurity radiation is a significant power loss mechanism in $2 X I I B$, this must be due primarily to impurities in the end fans.

However, there are broader implications of this result. The power loss from $2 X I I B$ is about $10 \mathrm{~W} \mathrm{~cm}^{-3}$. While this is small compared to the $280 \mathrm{~W} \mathrm{~cm}^{-3}$ deposited by the neutral beams*, it is not small in the context of mirror experiments with larger volumes and large surface areas. The $7 M X$ plasma volume is about $1.7 \times 10^{6} \mathrm{~cm}^{-3} .24$ A $10 \mathrm{H}$ $\mathrm{Cm}^{-3}$ power loss on $\mathrm{TMX}$ would impty $17 \mathrm{MW}$ of impurity power loss, whereas the planned power deposition is $<5 \mathrm{MW}$. On the other hand, transport effects and increases in $\mathrm{T}_{e}$ are expected to significantly decrease the power loss from the large solenoidal volume of TMX. It is

*For comparison, a typical tokamak might deposit $1 \mathrm{~W} \mathrm{~cm}^{-3}$ and radiate $0.3 \mathrm{~W} \mathrm{~cm}^{-3} .14$ 
clear that impurities will become a major consideration in the design and operation of large mirror experiments.

\section{LIGHT IMPURITY RESULTS}

The methods described in Section II were used to determine the concentrations and lifetimes of the light impurities in 2y.11B. Table VI-1 presents these results; they are discussed below. Table VI-1 bears out the initial conclusion of Section III Lnat oxygen is the dominant fmpurity in 2XIIB. The scattering of deuterium off this oxygen and other similar plasma effects have not yet been considered.

The calculation of impurity densities fnvolved a sum over all ionization states, using Eq. (8) of Section II. These calculations show that the 2XIIB plasma is mostly deuterium, but that it has a higher concentration of impurities than a clean tokamak. ${ }^{18}$ These results imply that

$$
Z_{\text {eff }}=\sum N_{i} Z_{i}^{2} / H_{i} Z_{i}
$$

is $1.85 \pm 0.4$ at the center of $2 X I I B$. The principal uncertainties in these estimated densities are the populations of unmeasured Helium-like ionization states, such as 0 VII. These populations were estimated by the balance of ionization into and charge-exchange out of these states.

The Iffetime of oxygen was estimated by using Eq. (II-5) and calculating the volume rate of input from 0 IV to $0 V$ (Eq. II-6). The result was $280 \mathrm{\mu s}$. This is comparable to the Spitzer drag time for 0 $V I$ at the center of the plasma of 10/24.77, which was $260 \mathrm{Hs}$. ( $\mathrm{T}_{\mathrm{e}}$ was $150 \mathrm{eV}$ and $\hat{n}_{\mathrm{e}}$ was $9.4 \times 10^{13} \mathrm{~cm}^{-3}$ ) This suggests that the oxygen is mirror confined. 
TABLE VI -1

LIGHT IMPURITIES IN 2XIIB

$\begin{array}{cc}\text { Species Concentration } & \text { Lifetime } \\ \text { at Plasma } & \text { Current to } \\ \text { Center } & \text { Central } \\ & \text { Plasma }\end{array}$

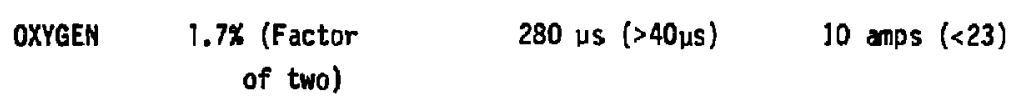

$\begin{array}{ccc}\text { NITROGEN } & 0.4 \% \text { (Factor } \\ \text { of three) } & 50 \mu s(>25 \mu \mathrm{s}) & 5 \text { amps (<25) }\end{array}$

CARBON $\quad 0.3 \% \pm 0.2 \% \quad 40 \mu \mathrm{s}(>13 \mu \mathrm{s}) \quad 2$ amps $(<10)$ 
As shown below, estimates of $\tau_{c}$ based on reasonable models of the 2 XIIB potential imply that low energy impurities will leave the central plasma in less than $20 \mu \mathrm{s}$, during which time most oxygen jons should not even become 0 VI. It appears that the oxygen in 2XIIB is either injected with a high transverse velocity or rapidly heated to become mirror confined.

The ejection time for a cold impurity ion depends on the plasma potential. Once the cold impurity has gone a significant distance, such as $10 \mathrm{~cm}$, it should not return to the midplane as it cannot climb the potential hill and is not mirror trapped. If one considers a $Z=1$ oxygen ion accelerated by a 100 volt potential declining uniformly from the midplane to the mirror at $75 \mathrm{~cm}$, one finds it travels $15 \mathrm{~cm}$ in 14 HS. A better model describes the variation in $\phi$ according to the Boltzmann relation and the measured plasma scale length:

$$
\hat{n}_{e} e^{-z^{2} / b^{2}}=\hat{n}_{e} e^{-q \phi / k T}
$$

Here $z$ is the dimensfon along the magnetic field and $b$ is the $1 / e$ halfwidth of the axial plasma density profile. This way, taking the electric field

$$
E=\frac{d \phi}{d z} \text {. }
$$

ane finds that a thermal $Z=1$ impurity trave $1 \mathrm{~s} 16 \mathrm{~cm}$ in $20 \mu \mathrm{s}$. These results support the conclusion stated above.

The total oxygen entering the central plasma was computed by using Eq. (11) of Section II. This current app] ies to that oxygen which contributes significantly to the higher oxygen ionization states. If cold oxygen was impinging on the plasma surface and was being rapidly lost, these 
measurements would not reveal it. A detailed study of 0 II emissions will be necessary to evaluate that possibility.

The nitrogen and carbon densities and currents were evaluated by the same techniques as those of oxygen. Limits on unobserved states ( $N$ IV,N VI, $C V)$ were determined by considering ionization and charge exchange rates into and out of observed ionization states. The nitrogen and carbon lifetimes were computed using the simpler method of Eq. II-(7), which required less data. These estimates do not Indicate whether the nitrogen and carbon are mirror confined, or both and particularly carbon are low energy and short-1ived.

\section{LYMAN ALPHA MEASUREMENTS}

A spectral study of the Lyman alpha deuterium line was also performed on October 25 and 28, 1977. The observed line profile is presented in Fig. VII-1, and Fig. VII-2 shows the time development of the entire line. This experiment may be considered a prototype demonstration of a Lyman alpha diagnostic to measure neutral deuterium velocity distributions. Although the two angstrom resolution used and the shot to shot variation of the 2XIIB plasma preclude the observation of fine details of the Lyman alpha spectrum, the observed profile is consistent with knowledge of the neutral deuterium in 2XI1B obtained from other diagnostics. The basic analysis of this profile is described below.

The full width at half maximum (FWHM) of the observed line profile is 4.5 $\AA$. It is peaked in the center and has broad wings. A maxwellian profile with several keV energy can be fit to the data. However, this is not reasonable, given tie presence of a large deuterium flux in the neutra? beans, wich are at angles close to $90^{\circ}$ to the line of sight of the spectrometer. A more subtle analysis is necessary, as follows. 


\section{LYMAN ALPFA SPECTRAL PROFILE}

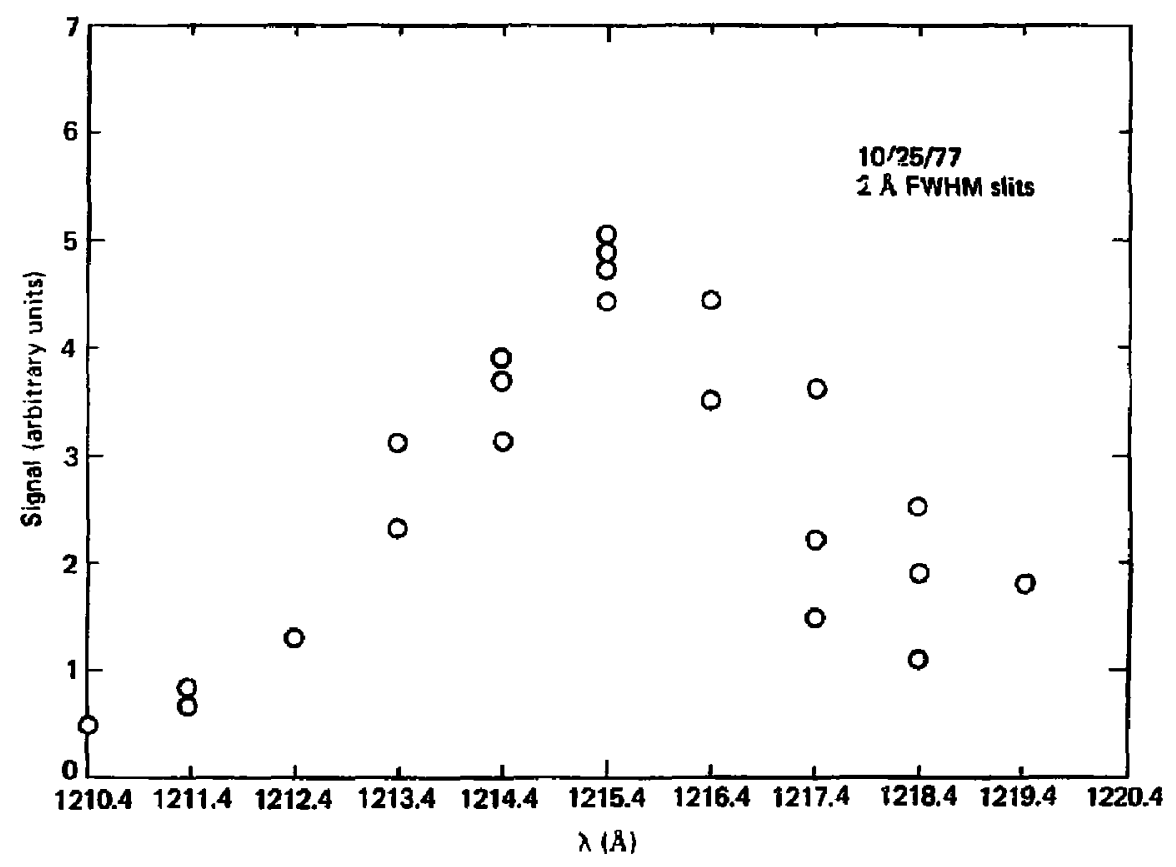

Figure VII-1. The observed spectral profile of Lyman alpha.

Each data point is from a single shot. 
LYWAN ALPHA TIIE DEVELOPFETT

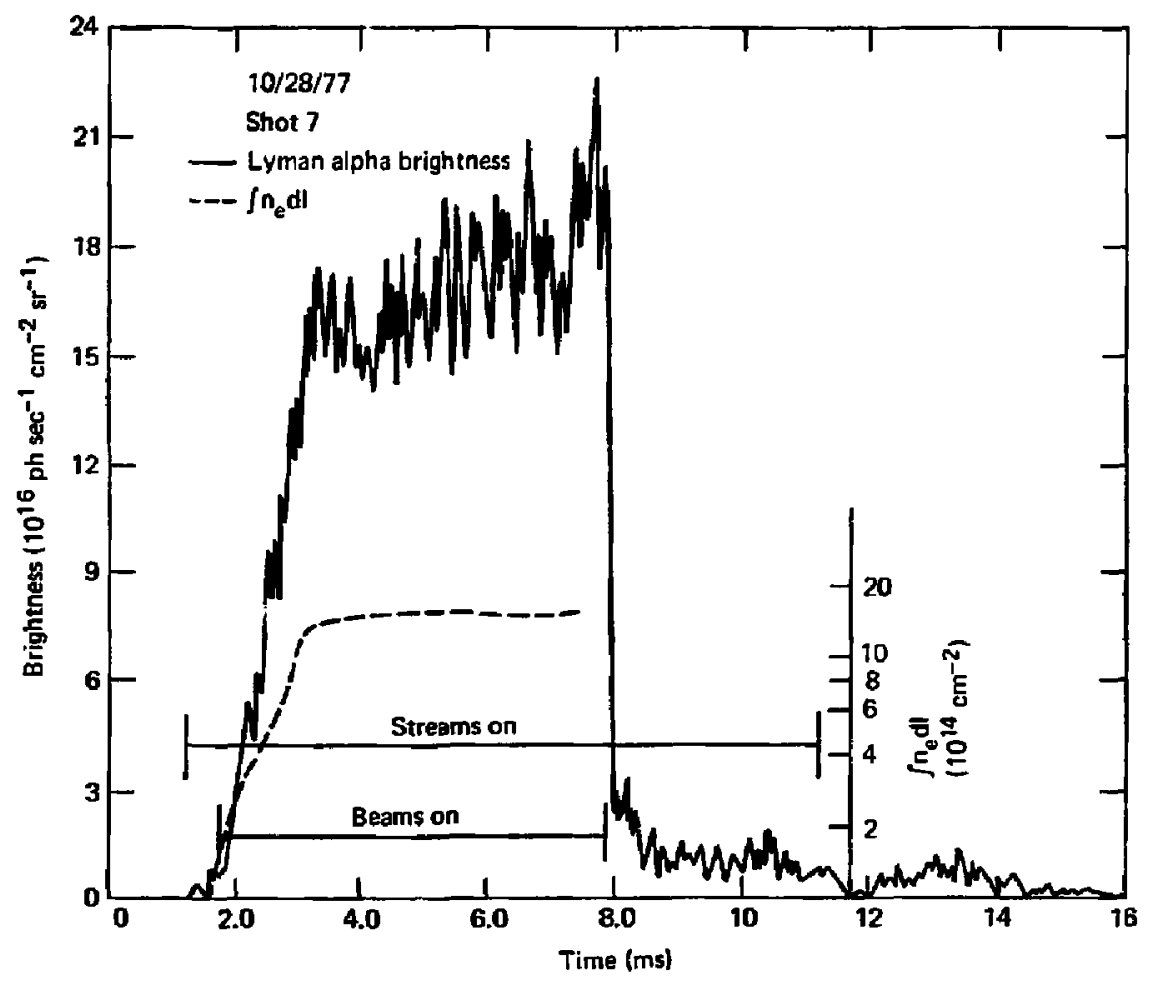

Figure VII-2. The Lyman alpha time development. Wide slits include the entire line. 
The neutral deuterium in $2 X I I B$ can be divided into three groups. There is a large charge exchange flux leaving the plasma, which is azimuthally symmetric. This flux results in a broad flat line profile (because of the quite non-maxwellian $2 X I$ IB velocity distribution), with an 8 A FWH. Secondly, there is a small cold gas flux to the surface of the plasma, producing a triangular profile at the peak of the line. Finally, the untrapped neutral beam particles produce an uneven and possible hollow profile, which is about 4.4 A FWHM, because the beam aiming is not quite perpendicular $\left(\sim \pm 22^{\circ}\right)$ to the spectrometer. Table VII-1 summarizes the Lyman alpha analysis, indicating the brightnesses of the various components of the line, as shown in Fig. VII-3. One concludes that these Lyman alpha observations are consistent with other knowledge of 2 XIIB.

Data from the center of the Lyman alpha profile suggests a cold gas brightness of about $1 \times 10^{16}$, as this signal remains after the beans have been turned off, eliminating the high energy neutral sources. Modifying Eq. II-(13) for the flux impinging on a piasma surface to include charge exchange,

$$
\Gamma=\frac{2 \pi B\left(\langle\sigma v\rangle^{i o n}+\langle\sigma v\rangle^{c x}\right)}{\langle\sigma v\rangle^{e x}} .
$$

For this brightness, $\Gamma \sim 2.4 \times 10^{17} \mathrm{~cm}^{-2} \mathrm{sec}^{-1}$. Assuming a $27 \mathrm{~cm}$ long $8 \mathrm{~cm}$ radius plasma, the cold neutral current is about $3 \times 10^{20} \mathrm{sec}^{-1}$, or about 50 amps at $8 \mathrm{~ms}$.

If this cold neutral current is charge-exchanging on hot plasma ions, the power loss by this mechanism must be large. Such power loss could be as high as 50 amps of $10 \mathrm{keV}$ ions, or $0.5 \mathrm{MH}$, which is one third the power input to $2 X I I B$. In that event, the $2 X I 1 B$ plasma might not sustain itself. One concludes that these measurements are consistent with the existence of a 
Table VII-1

LYMAN ALPHA STUDY

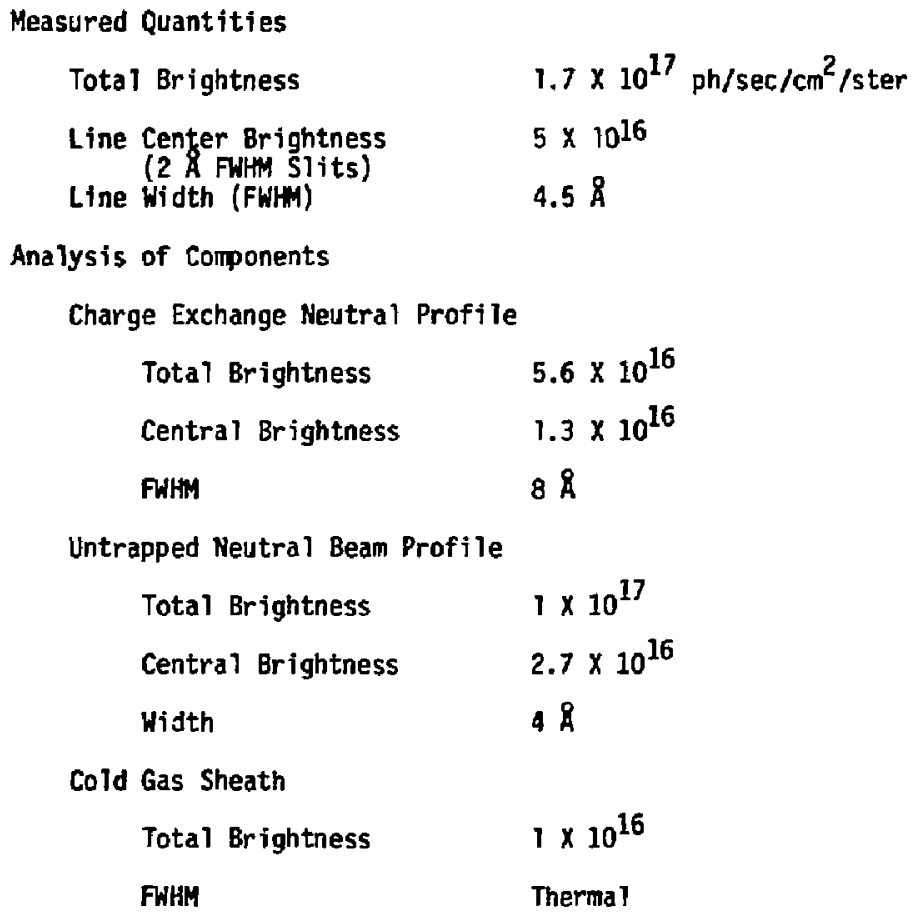

Table showing analysis of lyman alpha profile. Central brightness and all data were taken with a 2 \& FWM instrument transmission. 
ESTIIATEI COPPOSITIOI OF LYIAN ALPHA SPECTRAL PRDFILE

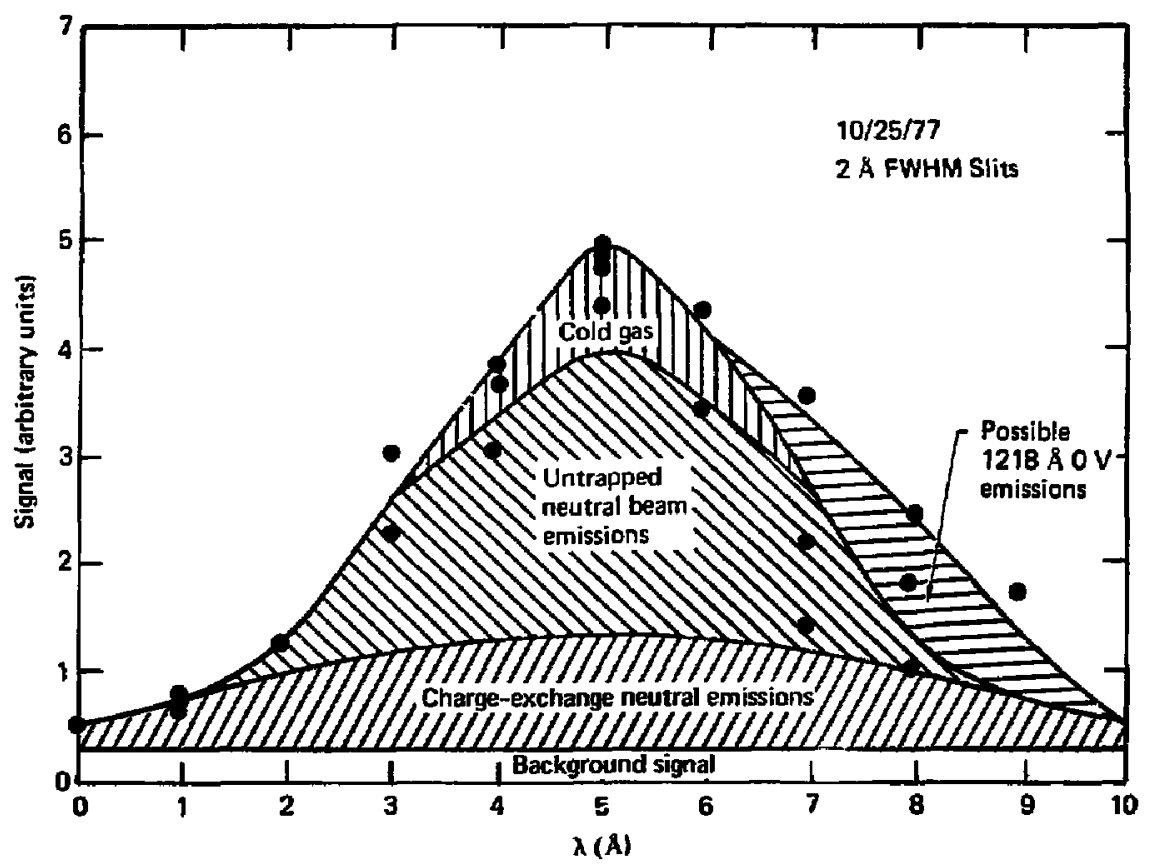

Figure VII-3. The observed spectral profile of Lyman alpha, with estimates of the contributions from various sources. 
shielding mechanism, ${ }^{25}$ which may consist of accumulated cold gas ions or of plasma ions which diffuse more slowly than they coor.

The Lyman alpha profile is observed to be asymetric. Examination of the data reveals characteristics in the time dependence on the red side of the line which match those of ov $630 \AA$. It is speculated on this basis that the ov $1218.4 \&$ line, a spin-forbidden intercombination transition, may interfere with the 1218 \& Lyman alpha signal. This would be correct if the $1218 \AA$ line were about $10^{-2}$ times as bright as the 630 \& ine.

\section{CONCLUSION}

The major results of this study of $2 X I I B$ are the determination of the principal impurity species and the measurement of the large brightnesses of their EUV emissions. Estimates of impurity power loss, confinement times, and concentrations were also obtainec. Table VIII-1 summarizes these results. These measurements have shown that while 2XIIB has impurity radiation density much larger than that of typical tokamaks, the relative power loss due to impurities in the central 2XIIB plasma is comparable to or less than that of the cleanest tok amaks.

The Lyman alpha study was consistent with other knowledge of the 2XIIB plasma. A large cold neutral flux is indicated; this is consistent with the hypothesis of a shielding mechanism protecting the 2XIIB hot ions from charge-exchange on cold gas.

The oxygen confinement time was estimated from the data to be longer than the ejection time for cold impurities affected by the ambipolar potential. This may be because the oxygen is beam injected, or because it is othermise heated and confined. This and other issues are being investigated in the continuing EUV study of the 2XIIB plasma. 
Table VIII-1

Summary of 2XIIB EUV Results

Impurities Identified

Oxygen, Nitrogen, Carbon Titanium

Central Plasma Light

Impurities: Density

$3 \%$

Power Loss*

$60 \mathrm{kH}$ (4\% of input)

0 VI $1032 \AA$

Brightness

$2 \times 10^{18}$ ph sec $\mathrm{cm}^{-2} \mathrm{sr}^{-1}$

$Z_{\text {eff }}$

T.9

Dominant Impurity

axygen (2x)

Lyman Alpha Brightness

$1.5 \times 10^{17} \mathrm{ph} \mathrm{sec}^{-1} \mathrm{~cm}^{-2} \mathrm{sr}^{-1}$

Cold Neutral Current

50 amps at $8 \mathrm{~ms}$

tRadiation from the end fan regions is not included. 


\section{ACKHOHLEDGEMENTS}

This work was supported by the U.S. Department of Energy through contracts H-7405-ENG-48 at the Lairence Livermore Laboratory and EY76-S-02-2711 at the Johns Hopkins University.

The authors wish to acknowledge the assistance of the sctentific and technica? staff of 2XI18; neither this data nor our understanding of it would have existed without their help. 
1. Coensgen et a1., Phys. Rev. Let. 35, 22, 1501 (1976).

2. Ohkawa, Nuc1. Fus. 10, 185 (1970).

3. Magee et a1., LASL Report LA-6691-MS.

4. Mattjoli, EUR-CEA-FC-761 (1975).

5. Davis, JQSRT 14, 549 (1974).

6. Davis et al., JQSRT 15, 1145 (1975).

7. Davis, MRL Report $\$ 33$.

8. Lotz, Astroph. J., Supplement 128, Vo 1. 14, May 1967, pp. 207-238.

9. Gardner, L.D., Ph,.. D. Thesis, Yale University, 1978.

10. Crandal1, O.H., private communication.

11. Barnett et al., ORNL Report 5206, Vol. 1.

12. Spitzer, Physics of Fully Ionized Gases, Second edition, wiley, 1962.

13. Futch et a1., Plasma Physics 14, 211 (1972) Eq. (44).

14. Equipe TFR, Nuc]. Fus. 15, 1053 (1975).

15. Terry et a1., JHU Technical Report co0-2711-3 (1977).

16. Coensgen et a1., LLL Internal Report UCID-17037 (1976).

17. Soloman and Ederer, Appl, Opt. 14, 1029 (1975).

18. Terry et a1., Nucl. Fus. 18, 485 (1978).

19. Simonen et a1, Nucl. Fus. 15, 813 (1975).

20. Terry, J.L., Ph. D. Thesis, Johns Hopkins University, 1978.

21. Elton, Atomic Processes, in Methods of Experimental Physics, Vol. 9-Part A, ed. by Griem and Lovberg.

22. Heise at a1., NBS report NSROS-NBS 4, VoT. 1.

23. Terry, J.L. private communication.

24. Coensgen et aT., LLL-Prop-148.

25. Stallard et al., LLL preprint UCRL-78741. 